\title{
An empirical taxonomy of visual illusions
}

\author{
STANLEY COREN \\ University of British Columtia \\ Vancouver, British Columbia, Canada V6T IW5 \\ JOAN S. GIRGUS \\ The City College of the City University of New York, New York 10010 \\ HOWARD ERLICHMAN \\ The Graduate Centre of the City University of New York, New York, New York 10036 \\ and \\ A. RALPH HAKSTIAN \\ University of British Columbia, Vancouver, British Columbia, Canada V6T IW5

\begin{abstract}
A classification system for visual-geometric illusions, based upon the interrelationships between behavioral responses to various distortions was created. Forty-five illusion configurations were presented to 221 observers. Factor analysis revealed that there are five classes of illusions. A secondorder analysis revealed that visual distortions are ultimately reducible to two global types of distortions: illusions of extent and illusions of shape or direction.
\end{abstract}

In the one hundred and twenty years since Oppel (1855) published the first systematic studies on visual geometric illusions, nearly a thousand papers have appeared that deal with distortions evoked by simple two-dimensional patterns of lines. Despite this massive research effort, it is interesting to note that we have still not managed to establish a classification scheme or taxonomy that would allow sensible groupings of the many known distortions.

It is true that classification cannot, in and of itself, provide explanations; however, a meaningful classification system has often served as a catalyst for theoretical advances in other areas of scientific endeavor. The classification systems of Mendelyev, in chemistry, and Linnaeus, in biology, immediately come to mind in this regard. Often, as in the above cases, successful taxonomies are based on descriptive categories, and the appropriate underlying mechanisms are often not isolated until many years after the classification scheme has been generally accepted as being useful. A classic example of this occurred shortly after 1816, when Thompson classified archeological finds according to the materials that were predominant in sets of artifacts, thus defining three separate eras that he designated as the stone, bronze, and iron ages. Only much later was this classification

We would like to acknowledge the assistance of Rosalind Wu, Jeanette Spero, Lucille Spivak, Louis Citron, Kathleen Moody, Cynthia Weinman, Gerald Marks, and Eva Kerzner in the collection and analysis of data. This research was supported in part by grants from the National Research Council of Canada (A9783) and the National Science Foundation (74-18599). scheme confirmed by stratographic evidence as indicating a real temporal sequence of cultures.

Attempts to classify visual geometric illusions date back to Wundt (1898), who attempted to divide illusions into groups on the basis of the nature of the illusory effects themselves. He suggested two classes of illusions: illusions of extent and illusions of direction. Unfortunately, Wundt himself was unhappy with this division, noting that there were several aberrant illusions that did not clearly fit into either class of distortion.

Most recent attempts at establishing typologies of illusions have followed Wundt's lead in relying upon the nature of the perceptual error to classify the configuration. For example, Oyama (1960) has proposed three major classifications. The first includes illusions of angle, direction, straightness, and curvature, a group roughly equivalent to Wundt's illusions of direction. The remaining two classes are produced by subdividing Wundt's illusions of extent into illusions of length and distance and illusions of size and area. Oyama was apparently dissatisfied with these classes, since he then divided each general group into subclasses which are so fine that virtually every major illusion pattern forms a separate entry.

Robinson (1972) follows a similar pattern. His first general grouping includes illusions of extent and area, with separate subheads for virtually all the major illusion figures. His second major grouping contains illusions in which the apparent direction of lines or size of angles are distorted. He then provides a third grouping, simply labeled "other illusions," 
in which we find the horizontal-vertical illusion and a variety of shape distortions.

Luckiesh (1922) used several criteria to divide illusions into five categories. The categories included (1) illusions of interrupted extent, which could be characterized by configurations such as the OppelKundt illusion (Figure 1J); (2) the effect of location in the visual field, which could be characterized by the horizontal-vertical illusion (Figure 1I); (3) illusions of contour, which include a number of MuellerLyer variants (Figure 2); (4) illusions of contrast, which include the Ebbinghaus and Delboeuf illusions (Figures 1E and 1D, respectively); and (5) illusions of perspective, which include variations of the Sander parallelogram (Figure 1L) and several Orbison configurations. Unfortunately, the basis for classification is not consistent. The first two categories refer to characteristics of the stimulus configuration itself, while the next two describe the direction of the distortion. The fifth category is completely different, referring to a particular theory of illusions. As Robinson (1972) has pointed out, such heterogeneity does not seem likely to lead to the extraction of underlying principles.

There have been two attempts to classify illusions according to a hypothesized underlying mechanism. Tausch (1954) suggests that all illusions are caused by a depth-processing mechanism that can lead to the inappropriate application of constancy scaling in a two-dimensional display. Since Tausch reduces all illusory distortions to this single mechanism, the problem of classification solves itself. An alternate classification scheme has been offered by Piaget (1969). He classifies illusions into two groups, based upon his theory of centrations, which he uses to explain changes in illusion magnitude with chronological age. His first category of illusions comprises those that decrease with age, while the second category comprises those that increase with age. Unfortunately, only the Ponzo (Figure 1F) and Ebbinghaus (IE) illusions seem to show consistent increases with chronological age (Leibowitz \& Judisch, 1967; Wapner \& Werner, 1957), while almost all other illusions seem to diminish in magnitude with age (Pick \& Pick, 1970). There are even some configurations that show an initial decrease followed by an increase, which also would provide some problems for this typology.

The problem of classification has resulted in much expenditure of experimental effort in attempting to assess relationships among configurations. Thus we find Fisher (1968) attempting to demonstrate experimentally that the Ponzo and Mueller-Lyer illusions fall within the same class, Girgus, Coren, and Agdern (1972) attempting to show that the Ebbinghaus and Delboeuf illusions are variants of one another, and, at the other extreme, experimenters such as Erlebacher and Sekuler (1969) attempting to show that the over-and underestimation portions of the Mueller-Lyer are separate illusory distortions, Quina and Pollack (1972) attempting to differentiate the over- and underestimation portions of the Ponzo illusion into separate categories, and Girgus and Coren (Note 1) attempting to separate experimentally all over- and underestimation configurations into separate illusory categories. These attempts to establish relationships among the various illusory configurations experimentally have not led us very far, since they deal with only limited sets of configurations, and the overall degree of correlation among the various illusory patterns is not yet well enough established to provide any meaningful base line against which to assess actual degrees of relationship. There are procedures, however, that may allow us to reasonably assess the interrelations among illusions, and thus empirically to establish a useful classification scheme.

Let us consider a hypothetical state of events that might lead us toward a behaviorally meaningful typology. Suppose that there are two underlying illusion mechanisms. For convenience, we will simply call them A and B. It would seem reasonable to classify illusions that are predominantly caused by mechanism $A$ into one group and those that are predominantly caused by mechanism $B$ into another. Of course, if both mechanisms are operating in any one configuration, there will be some interaction between the classes; however, segregation of illusion configurations into groups according to their dominant mechanisms still provides a behaviorally significant division. The pragmatic difficulty that arises in creating such a typology lies in the fact that we must first be able to define the underlying mechanisms. If the specific mechanisms are not correctly identified, the classification scheme is doomed to failure, and unfortunately we do not as yet know all the appropriate mechanisms involved in the formation of visual illusions. Suppose, however, that a particular individual is highly responsive to mechanism $\mathrm{A}$ and only weakly responsive to mechanism $B$. In testing such an individual, we would expect him to show sizable illusory distortions for configurations that are predominantly dependent upon mechanism $\mathrm{A}$ and only small illusory distortions for configurations predominantly dependent upon mechanism $B$. An individual who is more responsive to mechanism B than mechanism A should produce the opposite set of results. Notice that we are merely offering the suggestion that illusory magnitude covaries as a function of the strength of the underlying mechanism; yet this line of analysis suggests a means of producing a classification system. If there are large 

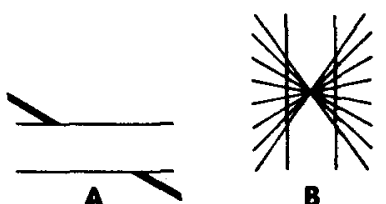

B
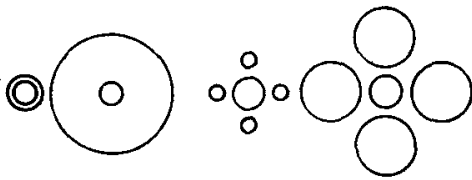

E

D

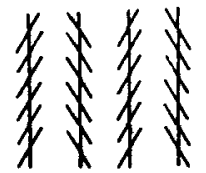

C

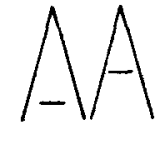

F

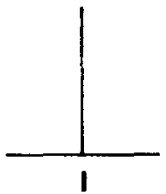

H

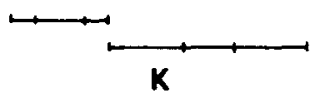

l
Figure 1. Variants of classical illusions in the stimulus set: the Poggendorff (A); Wundt (B); Zoellner (C): Delboeuf (D); Ebbinghaus (E); Ponzo (F); Jastrow (G); Baldwin (H); horizontalvertical (I); Oppel-Kundt (J); divided line (K), and Sander parallelogram (L).

individual differences in the magnitude of visual geometric illusions, and if these differences depend upon variations in an observer's sensitivity to a given mechanism or his propensity toward the use of a given perceptual strategy, then we can group illusions together on the basis of those distortions that tend to covary in magnitude, even though we have not specifically identified the underlying mechanisms or strategies. The experiment reported below describes an attempt to create an empirical taxonomy of illusions along these lines.

\section{METHOD}

\section{Stimuli}

Twelve of the most common classical illusion configurations were selected for inclusion. These included the Poggendorff (Figure 1A), Wundt (1B), Zoellner (1C), Delboeuf (1D), Ebbinghaus (1E), Ponzo (1F), Jastrow (1G), Baldwin (1H), horizontal-vertical (1I), Oppel-Kundt (1J), divided line (1K), and Sander parallelogram (IL). In addition, the 11 variants of the Mueller-Lyer illusion shown in Figure 2 were included. The purpose behind using a reasonably large set of classical illusion figures was to allow enough representative items so that meaningful groupings might emerge. The reason a large number of variants of a single illusion were included was to ascertain whether or not altered forms of a classical illusion, such as are commonly used in many experiments, actually represented the same

underlying mechanism or whether each variant represented a different illusion which ought to be classified under a separate heading.

Since we are interested in the covariation of illusion measures across a number of different configurations, it is important to make the required judgments as similar as possible so that any differences in responses will not artificially appear as a function of the measurement procedure. It was decided, therefore, to reduce all responses to the estimation of a horizontal linear extent of some part of the figure. For the Mueller-Lyer, Baldwin, and Oppel-Kundt configurations, the relevant extents are obvious. For the Ebbinghaus and Delboeuf illusions, observers were required to judge the diameter of the circles. Judgments of illusion magnitude for the Poggendorff, Wundt, Zoellner, and Jastrow illusions may also be reduced to judgments of linear extent by asking observers to judge the extents indicated as $\mathrm{X}$ or $\mathrm{Y}$ in Figure 3 for each configuration. Since all of the illusions used (except the Poggendorff) contain an over-and an underestimated dimension, each of these measures was taken separately. The 45 resulting illusion stimuli were each drawn on a separate $21 \times 27.5 \mathrm{~cm}$ page. In the lower left-hand quadrant of the page, there was a horizontal line on which the observer was asked to mark the apparent linear extent of some part of the figure. Coren and Girgus (1972) have shown that this technique provides results that are comparable in validity and reliability to those obtained by the method of adjustment. A small block of instructions and an illustrative diagram in the upper right-hand corner of each page indicated which extent was to be judged.

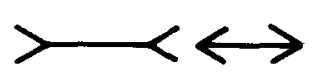

A
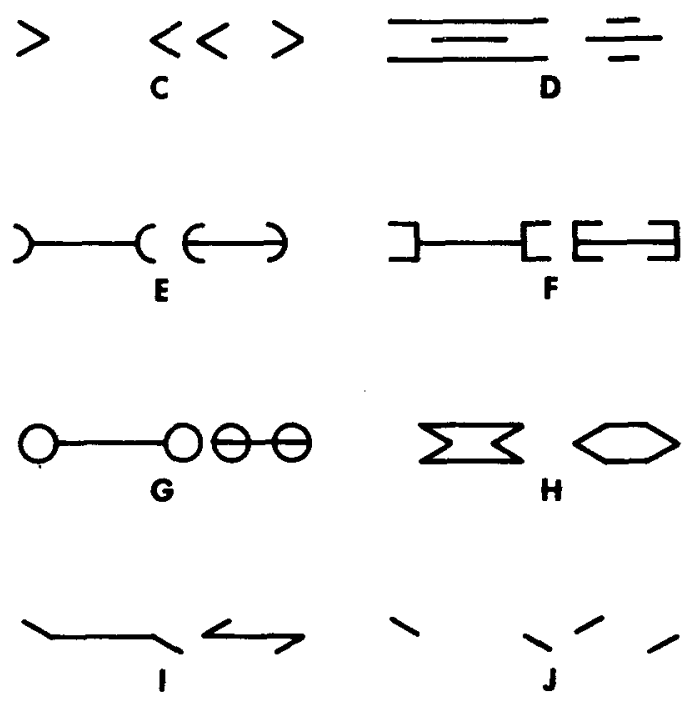

к $: \quad \because \dot{\bullet} \cdot$

Figure 2. Variants of the Mueller-Lyer illusions employed in this experiment: standard form (A); exploded (B): Piaget form (D): curved (E): box (F); circle (G): lozenge (H); Sanford form (I): minimal (J); Coren form (K). 

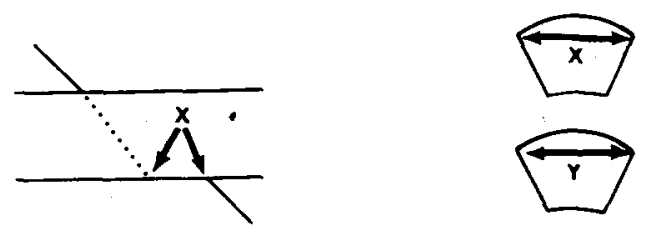

A

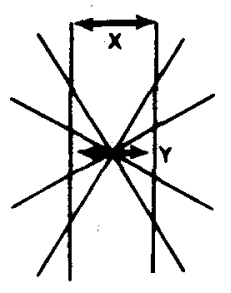

C

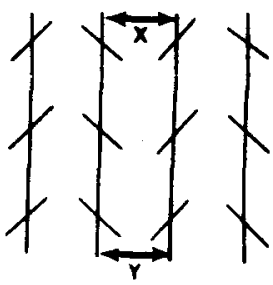

D

Figure 3. The horizontal linear extents that subjects were asked to estimate are indicated by $X$ or $Y$ for the Poggendorff (A), Jastrow (B), Wundı (C), and Zoellner (D) illusions.

\section{Procedure}

The illusion stimuli were assembled into booklets: Subjects received iwo such booklets, each of which contained the 45 illusion configurations in mixed order; thus, each subject made two independent judgments on each illusion extent. These two judgments were averaged for the purposes of statistical analysis.' Group administrations were utilized, and response rate was selfpaced, although subjects were urged to indicate their first impressions.

\section{Subjects}

The sample comprised 221 undergraduate volunteers from the City College of the City University of New York.

\section{RESULTS AND DISCUSSION}

A factor analytic technique was used to generate a classification scheme. First, the 45 variables were intercorrelated. In the resulting correlation matrix, virtually all the values were positive, a phenomenon which is frequently observed when dealing with measures of abilities or intelligence. In this study, only 31 of the 990 correlation coefficients were negative; the largest of these was -.14 , which, given a sample size of 221 , is not significantly different from 0 at the .01 level. Thus our analysis begins with a correlation matrix in which only $3 \%$ of the values are negative, and these are not significantly different from 0 . Such a positive manifold obviously indicates that, in general, illusion judgments are highly interrelated. It has been previously suggested that most visual geometric illusions are probably caused by

several mechanisms operating in the same direction to produce the final percept (Coren \& Girgus, 1973, 1974; Girgus \& Coren, 1973). Such multiple causation might very well lead to the positive manifold displayed in the correlation matrix. The high degree of interrelation among the illusion measures also seems to suggest that any factors that may be extracted will also be interrelated; hence, an oblique rather than an orthogonal solution seems to be indicated.

An oblique factor analytic solution was obtained by first performing an unweighted least-squares common-factor analysis on the 45 by 45 correlation matrix. Two criteria were used to determine the number of factors to be extracted. The first involved inspection of the eigen values of the correlation matrix, and the second involved the extraction of factors until the resultant pattern of factor loadings fulfilled the requirements of simplicity of structure (Guilford, 1954; Thurstone, 1947). Both procedures suggested the presence of five factors. The extracted factors were transformed by means of the generalized Harris-Kaiser (1964) procedure (see also Hakstian, 1970).

Table 1 shows the primary-factor matrix for the 45 illusion measures. The factor loadings above .40 have been underlined. For convenience, those segments that are usually overestimated in the various illusion configurations are indicated by $a+$ and those that are usually underestimated are indicated by a - next to the illusion name. Table 2 shows the intercorrelations among the extracted factors. It is interesting to note that the intercorrelations among the five factors shown in this table were all positive, thus again indicating the high degree of interrelatedness among illusion judgments obtained in the study.

Fortunately, the extracted factors seem to make sufficient sense in terms of what is known about illusions to provide a meaningful classification system. On the first factor, we find high loadings for the Poggendorff illusion and for both judgments of the Wundt and Zoellner illusions. In addition, the apparently longer segment of the Sander parallelogram and the apparently longer segment of the divided line illusion load on this factor. Ignoring the latter two for the moment, it is interesting to note that the Poggendorff, Wundt, and Zoellner illusions all contain intersecting line elements. These illusions are basically directional in nature, and can easily be explained by such relatively peripheral structural mechanisms as the blurring of the image in its passage through the crystalline lens and optic media (Chiang, 1968; Coren, 1969; Ward \& Coren, 1976) or lateral inhibitory interactions that displace contour loci (Békésy, 1967; Coren, 1970; Ganz, 1966) which serve 
Table 1

Factor Pattern for $\mathbf{4 5}$ Illusion Measures

\begin{tabular}{|c|c|c|c|c|c|c|}
\hline & I & II & III & IV & V & $h^{2}$ \\
\hline Poggendorff & .42 & .07 & .01 & .03 & .05 & .65 \\
\hline & .7 & .13 & -.05 & .03 & .09 & .50 \\
\hline undt - & .51 & .39 & .05 & -.20 & -.05 & .55 \\
\hline oellner + & .84 & .02 & -.28 & .16 & -.04 & .59 \\
\hline oellner - &.$\overline{74}$ & .11 & .01 & -.04 & .00 & .63 \\
\hline elboeuf - &.$- \overline{08}$ & .81 & .16 & -.19 & .13 & .59 \\
\hline Delboeuf + & -.10 & .62 & -.04 & .29 & -.10 & .23 \\
\hline bbinghaus - & .23 & .57 & .01 & .01 & .07 & .64 \\
\hline bbinghaus + & .15 &.$\overline{69}$ & -.25 & .07 & .04 & .51 \\
\hline Ponzo + & .07 & .62 & .09 & .08 & .04 & .77 \\
\hline onzo - & .05 &.$\overline{2}$ & .13 & -.03 & .66 & .61 \\
\hline Jastr & .08 & .49 & .15 & .27 &.$\overline{08}$ & .48 \\
\hline Jastr & .01 & $\overline{.38}$ & .07 & .29 & .15 & .54 \\
\hline Baldwin + & .02 & -.22 & .58 & .30 & .13 & .27 \\
\hline Baldwin - & .02 & .21 & .64 & .04 & -.02 & .68 \\
\hline Horiz- & .24 & -.06 & .67 & .00 & -.10 & .55 \\
\hline Horiz & .05 & .12 &.$\overline{36}$ & .38 & -.08 & .64 \\
\hline Oppel & .07 & .07 & .46 & .15 & .12 & .58 \\
\hline Oppel- & .04 & -.05 & $\overline{21}$ & .68 & -.05 & .06 \\
\hline$L+$ & .04 & -.03 & .69 & $\overline{.18}$ & -.16 & .52 \\
\hline ML - & -.12 & .16 &.$\overline{23}$ & .49 & .00 & .68 \\
\hline ed ML + & -.05 & -.12 & .51 &.$\overline{36}$ & .15 & .64 \\
\hline ML - & .12 & .13 & $\overline{.15}$ & .62 & .07 & .55 \\
\hline o Shaft ML + & -.07 & -.07 & .74 & .14 & .00 & .65 \\
\hline o Shaft ML - & .02 & .17 &.$\overline{14}$ & .50 & .06 & .67 \\
\hline Piaget ML + & .24 & -.03 & .70 &.$\overline{06}$ & .05 & .67 \\
\hline ML - & .05 & -.10 & $\overline{.11}$ & .68 & .20 & .54 \\
\hline $\operatorname{ved} \mathrm{ML}+$ & -.11 & .04 & .62 &.$\overline{24}$ & .19 & .60 \\
\hline Irved ML - & -.12 & .03 &.$\overline{11}$ & .59 & .30 & .66 \\
\hline Box ML + & -.10 & .11 & .59 & $\overline{.17}$ & -.02 & .65 \\
\hline Box ML - & -.19 & .1 & $\overline{.34}$ & .39 & -.04 & .66 \\
\hline Circle ML + & -.08 & -.07 & .64 & .16 & .19 & .57 \\
\hline Circle ML - & -.33 & .06 &.$\overline{49}$ & .31 & -.01 & .54 \\
\hline Lozenge ML + & -.15 & -.07 &.$\overline{70}$ & -.04 & .12 & .62 \\
\hline L - & .02 & .00 & $-\overline{23}$ & .96 & .01 & .59 \\
\hline $\mathbf{M L}+$ & .07 & .13 & .81 & $-\overline{\mathbf{0} 3}$ & -.02 & .52 \\
\hline $\mathbf{M L}-$ & .05 & .02 & $\overline{.05}$ & .62 & .27 & .66 \\
\hline ML + & .03 & .12 & .49 & .28 & .25 & .72 \\
\hline ML - & -.07 & .22 & .01 & .63 & .21 & .66 \\
\hline ML + & -.03 & .02 & .85 & .20 & .04 & .56 \\
\hline ML - & .22 & .00 & .19 & .56 & -.03 & .50 \\
\hline Divided Line + & .45 & .09 & .13 & $\overline{.05}$ & .30 & .52 \\
\hline Divided Line - & .02 & .16 & -.06 & .15 & .69 & .61 \\
\hline Sander Parallelogram + & .56 & -.09 & .28 & .27 & $-\overline{10}$ & .59 \\
\hline Sander Parallelogram - & .18 & -.06 & .00 & -.08 & .16 & .48 \\
\hline Factor Variance & 3.57 & 3.99 & 9.04 & 7.43 & 1.84 & \\
\hline
\end{tabular}

to displace the vertex of angles so as to render them apparently more obtuse. Why one segment of the Sander parallelogram and one segment of the divided line illusion should also load on this factor is not immediately clear. However, the other five high factor pattern loadings do suggest that Factor I involved structural, contour interactive mechanisms.

On the second factor we find high factor pattern coefficients for both halves of the Delboeuf and Ebbinghaus illusions, the apparently longer segment of the Ponzo illusion, and the apparently larger component of the Jastrow illusion. The apparently smaller portion of the Jastrow illusion just misses the criterion cutoff with a factor loading of 0.38 .
This factor appears to be quite homogeneous, and readily suggests a theoretical interpretation. All the illusion forms with high loadings on this factor seem to involve some sort of cognitive contrast effect in which the apparent difference in the size of the central test element and adjacent or surrounding elements is accentuated. Such accentuation of clearly perceived differences has been recognized since the time of Helmholtz $(1856,1860,1866 / 1962)$, who referred to it as "size contrast." That these illusory effects are clearly dependent upon cognitive information processing strategies has been demonstrated a number of times (Coren, 1971; Coren \& Miller, 1974) and several quantitative models based upon such cognitive processes have been of fered (Helson, 1964; Massaro \& Anderson, 1971; Restle, 1971).

Factors III and IV are strongly interrelated $(r=0.73)$. Given the fact that most of the high loadings that appear on these two factors represent variants of the Mueller-Lyer illusion, this is perhaps not surprising.

Factor III shows high factor coefficients for all 11 variants of the apparently longer segment of the Mueller-Lyer illusion. The only apparently shorter segment that intrudes on this factor is the dumbbell version. In addition, both segments of the Baldwin illusion, which may easily be interpreted as a variant of the apparently longer segment of the Mueller-Lyer illusion with boxed ends, appear on this factor. It is important to note that this is not purely a MuellerLyer factor. The apparently longer segment of the horizontal-vertical illusion and the apparently longer segment of the Oppel-Kundt illusion also manifest high factor loadings. In sum, this factor contains, almost exclusively, illusory distortions involving overestimation of linear extent. The specific mechanism underlying this grouping is not immediately clear, since quite different mechanisms have usually been proposed to explain the Mueller-Lyer, horizontalvertical, and Oppel-Kundt illusions.

It is interesting to contrast Factor III with Factor IV. On Factor IV, we find 9 of the 11 apparently shorter variants of the Mueller-Lyer illusion loading significantly, with the remaining two apparently shorter variants (the variant with boxed ends and the dumbbell form) just missing the criterion with weightings of 0.39 and 0.31 , respectively. In addition, the apparently shorter segment of the Oppel-Kundt

Table 2

Correlation Matrix of Factors (PHI)

\begin{tabular}{llllll}
\hline & I & II & III & IV & V \\
\hline I & & .45 & .12 & .24 & .26 \\
II & .45 & & .32 & .48 & .38 \\
III & .12 & .32 & & .73 & .18 \\
IV & .24 & .48 & .73 & & .31 \\
V & .26 & .38 & .18 & .31 & \\
\hline
\end{tabular}


illusion appears on this factor, while the apparently shorter segment of the horizontal-vertical illusion just misses the cutoff with a factor loading of 0.38 . Thus, this factor seems to represent the set of distortions associated with underestimation of linear extents. It is not clear why Factors III and IV segregate themselves in this fashion. To be sure, the high correlation between them indicates similarities between the two classes. Since the two factors contain separate halves of the same distortions, they would be expected to manifest some relationship on purely logical grounds. To find that the apparently longer and the apparently shorter sections of the Mueller-Lyer load on separate factors is quite interesting, since it has been suggested previously, based upon the fact that the two halves of the Mueller-Lyer do not covary in the same fashion in response to certain parametric manipulations, that they may in fact be separate distortions (Erlebacher \& Sekuler, 1969). To find that the horizontal-vertical and Oppel-Kundt illusions separate in a similar fashion, along with the Mueller-Lyer illusion, is surprising, since no similar suggestions have been made to segregate these configurations on the basis of their over- and underestimation segments.

It is also interesting to note that Factors III and IV are not more highly correlated with Factor II. On the whole, Factor II also involves over- and underestimations, but the distortions in Factor II apparently result from a different mechanism than do the distortions in Factors III and IV. It may be important to note that all the illusions that load on Factor II, except for the Ponzo illusion, involve the estimation of area, whereas the distortions in Factors III and IV involve linear extents. This cannot, however, provide the only explanation for a difference between these factors, since there is no hint of a separation between the under- and overestimated segments of the illusions in Factor II. It seems likely that quite different mechanisms underlie the illusions represented in Factors II, III, and IV.

The most puzzling factor is Factor V, which shows only two significant factor pattern coefficients: the apparently shorter segment of the Ponzo illusion and the apparently shorter segment of the divided-line illusion. It is possible to view both of these figures as enclosed segments that occupy only a small portion of the total bounded space, which would suggest that we may be dealing with the comparison of an element to its global frame of reference. However, since so few configurations load here, interpretation is difficult. We did not use a large enough sample of frame-of-reference illusions in this study to assess this interpretation properly. It is somewhat comforting that this factor accounts for such a small portion of the variance, approxi- mately $1 / 2$ that of Factors $I$ and II and $1 / 5$ that of Factors III and IV.

Finally, it should be pointed out that only one of the $\mathbf{4 5}$ illusion forms used in this study failed to load significantly on any of the five factors described above. This is the apparently shorter segment of the Sander parallelogram, whose factor pattern coefficients ranged from 0.00 to 0.18 .

On the basis of these results, we may now tentatively offer a classification scheme that can be used as a taxonomy for illusory distortions. The labels and generalizations attached to each class must be considered as somewhat speculative; however, the groupings are based on actual behavioral data and therefore may well reflect common underlying causal mechanisms.

(1) Shape and direction illusions. This class of illusions is characterized by Factor I. This grouping predominantly includes distortions in apparent shape, parallelism, and colinearity, which seem to arise in patterns with numerous intersecting line elements. The underlying mechanism is presumably structural in nature, probably involving optical aberrations and lateral inhibitory influences. The Poggendorff, Wundt, and Zoellner illusions are characteristic of this class, and presumably other shape and directional distortions, such as the Orbison or the Jastrow-Lipps illusions, would also fall into the same calssification.

(2) Size contrast illusions. This classification, characterized by Factor II, represents those illusory distortions in which the apparent size of an element appears to be affected by the size of other elements that surround it, or form its context. Thus, the casual mechanism may involve the use of sharpening or leveling strategies, such as those that have been quantitatively described by Helson (1964) Massaro and Anderson (1971) and Restle (1971). These effects typically manifest themselves as cognitive contrast, where a target appears larger when surrounded by small elements or smaller when surrounded by large elements. The Delboeuf, Ebbinghaus, Jastrow, and Ponzo illusions are characteristic of the illusions on this factor. Illusions involving contrast between the size of angles would presumably also fall into this classification.

(3) Overestimation illusions. This classification is best characterized by Factor III. The illusions that show the highest loadings on this factor include all the apparently longer versions of the Mueller-Lyer illusion, both parts of the Baldwin illusion, the apparently longer segment of the horizontal-vertical illusion, and the apparently longer segment of the Oppel-Kundt illusion. It is hard to ascertain what underlying causal mechanism may be associated with all these figures. For many of them, a depth 
processing interpretation, such as that popularized by Gregory (1968), seems plausible; yet, for others, such as Coren's dot form or the minimal form of the Mueller-Lyer, such an interpretation seems difficult. Simple spatial averaging may play a part in the Baldwin and the Mueller-Lyer figures; however, the Oppel-Kundt and horizontal-vertical do not seem susceptible to this form of explanation. Thus, we are left with a grouping on the basis of the behavioral manifestation that does not immediately suggest any underlying mechanism but suggests a pattern of interrelationship.

(4) Underestimation illusions. This group of illusions is represented by Factor IV. Since it includes most of the apparently shorter segments of the MuellerLyer illusion, the apparently shorter segment of the Oppel-Kundt, and the horizontal-vertical illusions, it seems to be a factor that is the complement to Factor III, representing predominantly underestimations of linear extent. Again, no mechanism immediately suggests itself for this class of distortion. It should be kept in mind that the linear illusions of over- and underestimations seem to be highly correlated and only an oblique factor analytic solution was able to separate these dimensions; thus, it is likely that the underlying mechanisms for these two factors may be somewhat related.

(5) Frame of reference illusions. This classification, characterized by Factor V, is the most tentative. Both of the high factor loadings are characterized by line segments that are underestimated in length; however, this pair of illusory distortions does not apparently covary with those of Factor IV, as might be expected if they were simply illusions of linear underestimation. In some respects, it is possible to see a configurational identity between these patterns and the variants of the Mueller-Lyer in Factor III, since the inducing elements are appended to the ends of the element to be judged. However, the Factor III illusions are all illusions of overestimation and the illusions in this factor are all of underestimation. On purely logical grounds, one might expect that the Ponzo illusion and the divided-line illusions would fall into Factor II, since both seem to involve some sort of cognitive contrast effect. In fact, it is interesting to note that the highest intercorrelation between Factor $\mathrm{V}$ and the other four factors is with Factor II, the size contrast factor. We have tentatively identified this as a frame-ofreference factor. If this interpretation is correct, illusions like the rod-and-frame ought to fall into this classification. Further experimental investigation is clearly necessary to specify this grouping more clearly.

We have, thus, produced a classification scheme that contains five bins into which illusory distortions may be sorted. Two of these bins, that containing illusions of direction and shape and that involving size contrast, seem to suggest underlying mechanisms. Of the remaining three, two are highly related (illusions of over- and underestimation) and seem logical, although no underlying mechanism immediately suggests itself. The fifth and final bin must be seen as merely suggestive at this point.

\section{Higher Order Classification}

Despite the fact that the taxonomy we have derived seems to be meaningful, it is interesting to consider a further form of analysis that may allow a more global pattern of groupings to emerge. This may be done via a second-order factor solution in which the intercorrelations between the factors shown in Table 2 serve as the data. In order to find higher order, or metagroupings, the five-by-five matrix was factored by the unweighted least squares method. Two factors were adequate to explain the higher covariation. These two factors were transformed to an oblique simple structure using the Harris-Kaiser (1964) procedure. Finally, the primary factor pattern loadings of measured variables projected on the two second-order factors were computed using the Cattell-White (Cattell, 1965) method. Table 3 shows the resultant matrix, which contains the factor loadings for the 45 illusion variants on the two second-order factors. These factors are correlated, with $r=0.49$.

It is clear that Factor $\mathbf{A}$ includes both the over- and underestimation segments of almost all the variants of the Mueller-Lyer illusion, both segments of the horizontal-vertical illusion, both segments of the OppelKundt illusion, and one segment of the Baldwin and Sander illusions. This factor seems to involve nearly all the illusions of linear extent in the test set. Factor B in the second-order solution contains the Delboeuf, Ebbinghaus, Ponzo, divided line, Wundt, and Zoellner illusions, with the Jastrow illusion just missing the criterion value. All these distortions may be seen as involving some sort of cognitive contrast. We have already referred to the Delboeuf, Ebbinghaus, divided line, Ponzo, and Jastrow illusions as examples of size contrast in our discussion of Factor II. It is interesting to note, in this regard, that the various over- and underestimation portions of these configurations tend to load on Factor B with opposite signs. Such a result would be consistent with a contrast explanation. The Wundt and Zoellner illusions may load here as examples of direction contrast, as originally suggested by Helmholtz (1866/1962).

It is somewhat suggestive to consider the fact that Factor B deals predominantly with distortions involving area, shape, and direction, while Factor $A$ contains mostly distortions of linear extent. It may well be the case that the earlier investigators who grouped 
Table 3

High-Order Factor Pattern Loadings for 45 Illusion Measures

\begin{tabular}{|c|c|c|}
\hline & A & B \\
\hline Poggendorff & .00 & .34 \\
\hline Wundt + & .07 & .46 \\
\hline Wundt - & .20 & .61 \\
\hline Zuellner + & .00 &.$\overline{51}$ \\
\hline Zoellner - & .10 &.$\overline{54}$ \\
\hline Delboeuf - & -.11 & $--\overline{74}$ \\
\hline Delbocuf + & .26 & .51 \\
\hline Ebbinghaus - & .04 & .60 \\
\hline Ebbinghaus + & .18 &.$- \overline{60}$ \\
\hline Ponzo + & .05 & .50 \\
\hline Ponzo - & -.19 &.$- \overline{46}$ \\
\hline Jastrow + & .09 & $\overline{.34}$ \\
\hline Jastrow - & .36 & .37 \\
\hline Baldwin + & .18 & -.28 \\
\hline Baldwin- &.- .50 & -.11 \\
\hline Horiz-Vert + & .52 & .10 \\
\hline Horiz-Vert - & .62 & .10 \\
\hline Oppel-Kundt + & .52 & .13 \\
\hline Oppel-Kundt - & .78 & .09 \\
\hline Standard ML + & .72 & .04 \\
\hline Standard ML - & .62 & -.16 \\
\hline Exploded ML + &.$\overline{08}$ & -.25 \\
\hline Exploded ML - & .44 & -.06 \\
\hline No Shaft ML + &.$\overline{48}$ & -.16 \\
\hline No Shaft ML - & $\overline{.31}$ & -.06 \\
\hline Piaget ML + & .61 & .15 \\
\hline Piaget ML - & .71 & .26 \\
\hline Curved ML + &.$\overline{74}$ & -.04 \\
\hline Curved ML - &.$\overline{65}$ & .14 \\
\hline Box ML + & $\overline{65}$ & -.01 \\
\hline Box ML - & .65 & .02 \\
\hline Circli ML + & .68 & -.04 \\
\hline Circle ML - &.$\overline{68}$ & .01 \\
\hline Lozenge ML + & .57 & -.04 \\
\hline Lozenge ML - & .67 & .11 \\
\hline Sanfcrd ML + &.$\overline{61}$ & -.11 \\
\hline Sanferd ML - &.$- \overline{50}$ & .09 \\
\hline Minimul ML + &.$\overline{.64}$ & -.01 \\
\hline Minimal ML - &.$\overline{58}$ & .09 \\
\hline Curen ML + & .52 & -.03 \\
\hline Coren ML - & $\overline{.63}$ & .17 \\
\hline Divided Line + & $-\overline{13}$ & -.48 \\
\hline Divided Line - & .24 & $\overline{.45}$ \\
\hline Sander Parallelogram + &.- .41 & $-\overline{\mathbf{3 4}}$ \\
\hline Sander Parallelogram - & $-\overline{10}$ & -.01 \\
\hline
\end{tabular}

illusions into two categories, one involving illusions of extent and the other illusions of shape, may well have been on the right track, at least in terms of the two metafactors obtained in this study. Such a global analysis is suggestive; although it does not permit the finer classification that the basic solution allows.

In conclusion, we may describe our data as demonstrating that a taxonomy of visual illusions may be created based upon behavioral data. The superordinate division of illusory distortions seems to be in two general classes. The first contains illusions involving distortions of linear extent, while the second contains illusions of area, shape, and direction. However, complete classification of the illusions in this study requires a subdivision of the set of stimuli into at least five classes. The first of these classes seems predominantly structural in nature and involves both shape and directional illusions. The second class seems to involve a size contrast mechanism. The third grouping contains a large number of illusions of linear overestimation, while the fourth class contains a large number of illusions involving linear underestimation. The fifth class mav involve frame-of-reference illusions.

It might be noted that it has been frequently suggested that most visual illusions are caused by multiplicity of mechanisms acting in concert (Coren \& Girgus, 1973, 1974; Girgus \& Coren, Note 1). Thus, while the classification scheme resulting from this study may suggest primary mechanisms for some of the test stimuli, we cannot begin to specify all the secondary mechanisms that may contribute to the various illusions. It seems likely that, for any given illusion, several mechanisms, in addition to that which might define the primary factor loadings, may contribute to the final percept.

It seems somewhat paradoxical that after 120 years of research we are only now offering a tentative classification scheme for visual illusions. This classification differs from others in that we have allowed the behavioral data to provide us with a taxonomy rather than forcing the groupings according to theoretical predispositions. It seems possible to extrapolate from these results to predict other illusions that should fall into particular classifications. Such class membership should be reflected by high intercorrelation with other members of that group.

Of course the underlying principles that produce the particular natural arrangement of the perceptual phenomena we have uncovered are not yet fully understood. There is some comfort to be derived from the fact that there is enough of an underlying order to allow us to organize illusion configurations in some manner that makes conceptual sense. Ultimately, one may hope that such a taxonomy will be used to reinterpret existing data and relationships among configurations, as well as serving as a guide to further research.

\section{REFERENCE NOTE}

1. Girgus, J. S., \& Coren, S. Contrast and assimilation illusions: Differences in plasticity. Paper presented at the meeting of the Psychonomic Society, Denver. November. 1975.

\section{REFERENCES}

Békésy, G. von. Sensory inhibition. Princeton, N.J: Princeton University Press, 1967.

Cattell. R. B. Higher order factor structures and reticular-vs-hierarchical formulae for their interpretation. In C. Banks and P. L. Broadhurst (Eds.), Studies in psychology. London: University of London Press, 1965. Pp. 223-266. 
Chiang, C. A new theory to explain geometrical illusions produced by crossing lines. Perception \& Psychophysics, 1968. 3, 174-176.

COREN, S. The influence of optical aberrations on the magnitude of the Poggendorff illusion. Perception \& Psychophysics, 1969, 6, 185-186.

Coren, S. Lateral inhibition and geometric illusions. Quarterly Journal of Experimental Psychology, 1970, 22, 274-278.

Coren, S. A size contrast illusion without physical size differences. American Journal of Psychology, 1971, 84, S65-566.

Coren, S., \& Girgus, J. S. A comparison of five methods of illusion measurement. Behaviour Research Methods \& Instrumentation, 1972, 4, 240-244.

Coren, S., \& Girgus, J. S. Visual spatial illusions: Many explanations. Science, 1973, 179. 5034

Coren. S., \& Girgus, J. S. Transfer of illusion decrement as a function of perceived similarity. Journal of Experimental Psychology, 1974, 102, 881-887.

Coren. S., \& Miller, J. Size contrast as a function of figural similarity. Perception \& Psychophysics, 1974, 16. 355-357.

Erlebacher, A., \& Sekuler, R. Explanation of the Mueller-Lyer illusion: Confusion theory reexamined. Journal of Experimental Psychology, 1969, 80, 462-467.

Fisher, G. H. Gradients of distortion seen in the context of the Ponzo illusion and other contours. Quarterly Journal of Experimental Psychology, 1968, 20. 212-217.

Ganz. L. Mechanism of the figural aftereffects. Psychological Review, 1966, 73, 128-150.

Grrgus, J. S., \& Coren, S. Peripheral and central components in formation of visual illusions. American Journal of Optometry and the Archives of the American Optometric Society, 1973, 50, 533-580.

Girgus, J. S., Coren, S., \& Agdern, M. The inter-relationship between the Ebbinghaus and the Delboeuf illusions. Journal of Experimental Psychology, 1972, 95, 453-455.

Gregory, R. L. Perceptual illusions and brain models. Proceedings of the Royal Society, 1968, Section B, 171, 279-296.

GuILford. J. Psychometric methods. New York: McGraw-Hill, 1954.

Hakstian, A. R. A computer programme for oblique factor transformation using the generalized Harris-Kaiser procedure. Educational and Psychological Measurement, 1970, 30, 703-705.

HARRIS, C. W., \& KaIser, H. F. Oblique factor analytic solutions by orthogonal transformations. Psychometrika, 1964. 29. 347-362.

Helmholtz, H. von. Treatise on physiological optics. Leipzig: Voss. Part I (1856), Part II (1860), Part III (1866). Republished: New York, Dover, 1962.

Helson. H. Adaptation-level theory: An experimental and systematic approach to behaviour. New York: Harper, 1964.
Leibowitz, H. W., \& Judisch, J. M. The relationship between age and the magnitude of the Ponzo illusion. American Journal of Psychology, 1967, 80, 105-109.

Luckiesh, M. Visual illusions. Princeton, N.J: Van Nostrand, 1922.

Massaro, D. W., \& Anderson, N. H. Judgmental model of the Ebbinghaus illusion. Journal of Experimental Psychology, $1971,81,147.151$

OpPEL, J. J. Über Geometrisch-optische Tauschungen. Jahresbericht der Frankfurt (Vereins), 1855, 55, 37-47.

OYAMA. T. Japanese studies of the so-called geometrical-optical illusions. Psychologia, 1960, 3, 7.20.

Piaget, J. The mechanisms and perception (Trans. G. N. Seagrim). New York: Basic Books, 1969.

Pick, H. L., \& Pick, A. B. Sensory and perceptual development. In B. Mussen (Ed.), Carmichael's manual of child psychology. New York: Wiley, 1970.

Quina. K., \& Pollack, R. H. Effects of test line position and age on the magnitude of the Ponzo illusion. Perception \& Psychophysics, 1972, 12, 253-256.

Restle, F. Visual illusion. In M. H. Apley (Ed.), Adaptationlevel theory. New York: Academic Press, 1971.

RoBinson, J. O. The psychology of visual illusion. London: Hutchinson, 1972.

Tausch, R. Optische Tauschungen als artisizielle Effekte der Gestaltungsprozesse von Grossen- und Formenkonstanz in der Natürlichen Raumwahrnehmung. Psychologische Forschung, 1954, 24, 299-348.

Thurstone, L. L. Multiple-factor analysis. Chicago: University of Chicago Press, 1947.

WAPNer, S., \& Werner, H. Perceptual development: An investigation within the framework of sensory-tonic field theory. Worcester: Clark University Press, 1957.

WARD, L. M., \& Coren, S. The effect of optically-induced blur on the magnitude of the Mueller-Lyer illusion. Bulletin of the Psychonomic Society, 1976, 7, 483-484.

WundT, W. Die geometrisch-optrischin Tauschungen. Abhandlungen der mathematisch physischen Classe der Koeniglischen Sacohsischen Gesellschaft der Wissenschaften. 1891, 24, 53-178.

\section{NOTE}

1. The mean intercorrelation between the two measures made on each configuration was 0.55 , with a standard deviation 0.18 . This corresponds to a mean reliability of 0.71 for this form of illusion measurement.

(Received for publication January 26, 1976; revision accepted April 28, 1976.) 